\title{
The interaction mechanism between the safety attitude and safety performance
}

\author{
Rui Zhang \\ Beijingjiaotong University \\ zhangrui0529@126.com
}

Keywords: safety attitude; safety performance; structural equation model

\begin{abstract}
. manufacturing's safety in production has an important influence on the development of economy and society. This paper will take the operators from a workshop as the research object and use the structural equation model to study the relationship and interaction mechanism between the safety attitude and safety performance based on the questionnaire survey. The research shows that the various dimensions of safety attitude will have a significant impact on the safety performance, and improving safety attitude is one of the important methods to improve safety performance from the theoretical support.
\end{abstract}

\section{Introduction}

Development of manufacturing industry encountered many problems. Its security situation is grim, bringing discordant factors for its booming manufacturing, affectting the social stability and development. With highlights safety issues, research on safety performance began to attract academic attention. Scholars began experimenting with a variety of effective ways to reduce human factors accidents, in order to enhance safety performance. However, scientific management in the security field, scholars at home and abroad study more secure environment, safety culture, safety behavior on affecting the safety performance, and relatively few studies on safety attitudes affecting safety performance, particularly in the typical industry is no empirical study. The article is trying to verify the relationship between employee safety attitudes and safety performance, providing new ideas for the security management of the manufacturing sector.

\section{Organization of the Text}

\section{Theory and hypothesis}

1) Safety Attitude

Attitude is the key subject of Social Psychology. As a psychological tendencies, attitudes will affect the way we behave on some extent, and consists of three elements: emotion, intention and awareness. The so-called security attitude means that employees held steady and general task dissociation and have the awareness of safety production.

2) Safety Performance

The definition of safety performance is quite different, but it can be roughly divided into three kinds: one is directly using the production safety accidents and their consequences to define safety performance; the second is to measure operating results of safety from the business reality performance; The third is using the accident situation and the reality of the performance of the enterprise to define safety performance.

Comprehensive studies about the definition of safety performance, the paper argues that the performance of the enterprise security is a comprehensive including incidents over a period of time, occupational injury cases and business security systems overall health. That the safety consequences of the accident data should be considered is not in doubt,because the ultimate goal of work safety is to reduce the accident rate, reduce accident losses and improve occupational safety and health. 
And due to the lag effect of the production characteristics of safety, the consequences of information security incidents do not reflect the reality of the situation alone and it can not play a preventive role in security. Its result is a variety of reasons at the same time.

3) Structural equation modeling

Structural equation modeling is a new statistical methods and ideas have a comprehensive use and improvement for confirmatory factor analysis, path analysis, regression and analysis of variance.

Has been widely used in management science, behavioral and social sciences, psychology and many other areas, it can handle a number of reasons as required relationships between multiple results, and can solve the problem of latent variables. it is an important tool for multivariate data analysis. Safety attitudes and safety performance are not directly observable variables, and these factors interact. Therefore, this paper adopt structural equation model to build the relationship between safety attitude and safety performance on the basis of a literature review, and propose theoretical assumptions and empirical test at a factory in order to enhance the safety performance of manufacturing direction.

4) assumption

Scholars have generally agreed that unsafe attitude of staff and unsafe behavior lead safety incidents to occur. DuPont believes the concept and attitudes of the employee will determine the safety performance of the enterprise. The Heinrch has put forward that an inappropriate attitude of staff is an important factor leading to unsafe behavior. Li Yan Zhang et al study shows that the attitude of motorcyclists on safety accident does have a direct impact. Chinese scholars have also found through empirical research on the production and management that employee safety attitudes and safe participation factor having a positive effect on the production and management. Can be inferred by the above discussion, employee safety attitudes have a significant impact on safety performance.

\section{Research methods:}

1) The study sample

The article regard the wokshop frontline workers as the research sample, the focus method is form of field research, combined with mailed questionnaires and forms of organization of meetings. A total of 200 questionnaires, 173 questionnaires were filled in the questionnaire and a large number of omissions fill in the blank and not serious to reject the entire questionnaire, and finally get 147 valid questionnaires pairing. The response rate was $86.5 \%$, of which the effective sample recovery was $73.5 \%$, the overall situation is still relatively good.

\section{2) Research Tools}

\section{a.Safety Attitude Scale}

This paper measures security attitudes by self scale. Which contains the security emotional safety attitude, safety awareness, safety intentions and a total of nine questions. The scale uses a fifth grade Likert scoring method, in a completely agree, somewhat do not agree, uncertain, somewhat agree, totally agree with other options, were given $1,2,3,4,5$ points.

b. Safety Attitude Scale

In this paper, safety performance measurement scale mainly compiled by the research reference Neal and Grif's made. Among them, the safety performance contains the security compliance and safety participation in two dimensions, a total of nine questions. The fifth grade Likert scale uses the same scoring method, in a completely agree, somewhat do not agree, uncertain, somewhat agree, totally agree with other options, were given $1,2,3,4,5$ points.

3) Demographics

Subjects of demographic data, including gender, age, education, work experience, marital status.

4) Statistical analysis

This paper adopt Amos7.0 and SPSS17.0 software for statistical analysis, including data processing, the use of scale reliability and validity, construct structural equation modeling approach to safety Performance. 


\section{Results}

1) Scale and Reliability

In this paper, cronbach's $\alpha$ to verify consistency of two scales contained in the variable, all cronbach's $\alpha$ coefficients are shown in Table 1. The results shown in the table can be obtained due to the consistency coefficient of each variable higher than 0.7 , indicating that this article used two scales have good reliability.

Table 1 Variable reliability analysis

\begin{tabular}{cccc}
\hline Variable & cronbach's $\alpha$ & Variable & cronbach's $\alpha$ \\
\hline Security emotion & 0.732 & Security compliance & 0.788 \\
Safety awareness & 0.775 & Safety Participation & 0.721 \\
Safe behavior & 0.751 & & \\
\hline
\end{tabular}

As used herein, in order to verify the scale factor structure, using Amos7.0 confirmatory factor analysis, the fit of each subscale shown in Table 2. Wherein X2 / df value between 1 to 3 is better; GFI, NFI, IFI, CFI, TLI ranging from 0 to 1 , if higher than 0.9 , then the better fit of the model; and RMSEA of the value is as small as possible, less than 0.05 indicates a very good fit. From Table 2 , article two scales X2 / df, GFI, NFI, IFI, CFI, TLI and RMSEA values were within normal range. Through the above analysis can be considered indicators, structural model fit as used herein, the scale is still relatively high.

Table 2 Scale common Fit Index

\begin{tabular}{cccccccc}
\hline Fit Index & $\mathrm{X}^{2} / \mathrm{df}$ & GFI & NFI & IFI & CFI & TLI & RMSEA \\
\hline Security attitude & 2.334 & 0.911 & 0.886 & 0.92 & 0.909 & 0.926 & 0.048 \\
Safety Performance & 2.447 & 0.902 & 0.923 & 0.955 & 0.901 & 0.921 & 0.052 \\
\hline
\end{tabular}

For the full depth exploration of attitudes and employee safety performance security relationship and interaction between the variables of each dimension, this paper establishes a security model, using Amos7.0 by maximum likelihood method to test the hypothesis that the theoretical model to give goodness index. Then, according to the model to modify the model calculation result, almost no effect on weed out assumptions, the final model, as shown in Figure 1

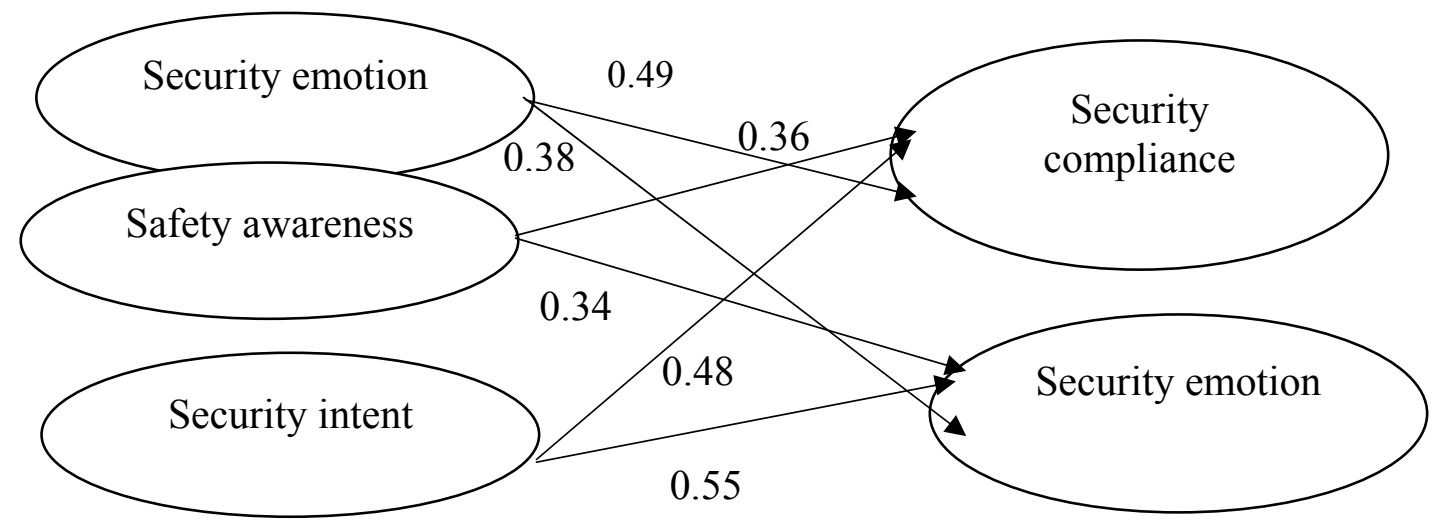

Figure 1 safety attitudes and safe performance of the fitted model

Table 3 safety attitudes and safety performance model fitting index

\begin{tabular}{ccccccccc}
\hline $\mathrm{X}^{2}$ & $\mathrm{df}$ & $\mathrm{X}^{2} / \mathrm{df}$ & GFI & NFI & IFI & CFI & TLI & RMSEA \\
\hline 629.126 & 267 & 2.356 & 0.911 & 0.927 & 0.915 & 0.952 & 0.934 & 0.042 \\
\hline
\end{tabular}


The final model fitting results are shown in Table 3, the absolute fit index X2 / df is 2.356, in the ideal range of 1 to 3 ; RMSEA 0.042, the standard is less than 0.05, indicating a small difference between the theoretical model and the saturation of the model; NFI is 0.927 , IFI is 0.915 , CFI and TLI 0.9340 .952 higher than 0.9 evaluation criteria. Fitting From the above analysis of the main model indicators can be seen fit to give the final article is fairly theoretical model is ideal.

2) Relation of the variables in the theoretical model

Standard path coefficients between safety attitudes and safety performance and its dimensions of this paper is shown in Figure 1.

From the figure, we can see that the security emotional produced a significant impact on safety compliance and safety participation.Its standardized path coefficient is 0.52 and 0.51 , respectively; and security awareness of security compliance and safety participation standardized path is 0.36 and 0.34 , which show that security awareness is an important factor affecting safety performance; security intention to security compliance and safety participation of the overall effects of the participation are 0.48 and 0.55 .

\section{Conclusions and Implications}

In the research of scholars, most related to the safety behavior, safety climate and the impact of safety culture on safety performance, and in many studies, it is assumed significantly affect safety attitudes towards safety performance has not been verified, or just get some verification. In the field of domestic security management, the interaction mechanism safety attitude and safety performance is rarely discussed, especially in the context of manufacturing, there has been no empirical research.

The principles described herein structural equation, field research, using survey data as a support for the relationship between safety attitudes and safety performance of a scientific study.It concluded as follows:

Security attitude can predict safety performance, safety attitudes significantly affect the safety and production. If staff can establish the correct safety attitude and develop good safety habits, it would have greatly contribution to job management. Manager's supportive attitude is a prerequisite for improved safety performance, and we shuould establish a healthy safety culture and improve safety performance, managers must influence and change the attitudes held by security staff.

\section{Summary}

In the research of scholars, most related to the safety behavior, safety climate and the impact of safety culture on safety performance, and in many studies, it is assumed significantly affect safety attitudes towards safety performance has not been verified, or just get some verification. In the field of domestic security management, the interaction mechanism safety attitude and safety performance is rarely discussed, especially in the context of manufacturing, there has been no empirical research.

The principles described herein structural equation, field research, using survey data as a support for the relationship between safety attitudes and safety performance of a scientific study.It concluded as follows:

Security attitude can predict safety performance, safety attitudes significantly affect the safety and production. If staff can establish the correct safety attitude and develop good safety habits, it would have greatly contribution to job management. Manager's supportive attitude is a prerequisite for improved safety performance, and we shuould establish a healthy safety culture and improve safety performance, managers must influence and change the attitudes held by security staff.

\section{References}

[1] Vaios Lappas,Nasir Adeli,Lourens Visagie,Juan Fernandez,Theodoros Theodorou,Willem Steyn,Matthew Perren. CubeSail: A low cost CubeSat based solar sail demonstration mission[J]. Advances Space Research, 2011,4811

[2] R. Keil,D. Risquez,F. van Leeuwen,A.G.A. Brown. An attitude model for the spacecraft of the ESA mission Gaia[J]. Acta Astronautica,2011,699 
[3] Matthias Gerner. The fuzzy logic of socialised attitudes in Liangshan Nuosu[J]. Journal of Pragmatics, 2010,4211

[4] Vaios Lappas,Nasir Adeli,Lourens Visagie,Juan Fernandez,Theodoros Theodorou,Willem Steyn,Matthew Perren. CubeSail: A low cost CubeSat based solar sail demonstration mission[J]. Advances in Space Research,2011, 4811

[5] Liusu Xia, Mei Qiang, Shen Bin, Zhang Chan Chan Safety Performance Review [J] China Safety Science, 2010,05: 131-139.(in chinese)

[6] Yang Shijun empirical analysis for construction enterprise safety culture and safety performance and comparative study of the relationship between [D]. Southwest Jiaotong University, 2013. (in chinese)

[7] Wang Jinfeng, Liu Zhenfeng, Feng Lijie, Feng Jian coal mine safety investment and safety performance of the system dynamics [J] Liaoning Technical University (Natural Science), 2011,02: 182-185. (in chinese) 